\title{
THE VELOCITIES OF INTRANETWORK AND NETWORK MAGNETIC FIELDS
}

\author{
H. WANG \\ Physics Department, New Jersey Institute of Technology, U.S.A. \\ F. TANG and H. ZIRIN \\ Big Bear Solar Observatory, California Institute of Technology, U.S.A. \\ and \\ J. WANG \\ Beijing Astronomical Observatory, China
}

(Received 12 May, 1995; in final form 21 September, 1995)

\begin{abstract}
We analyzed two sequences of quiet-Sun magnetograms obtained on June 4, 1992 and July 28, 1994. Both were observed during excellent seeing conditions such that the weak intranetwork (IN) fields are observed clearly during the entire periods. Using the local correlation tracking technique, we derived the horizontal velocity fields of IN and network magnetic fields. They consist of two components: (1) radial divergence flows which move $\mathrm{IN}$ fields from the network interior to the boundaries, and (2) lateral flows which move along the network boundaries and converge toward stronger magnetic elements. Furthermore, we constructed divergence maps based on horizonal velocities, which are a good representation of the vertical velocities of supergranules. For the June 4, 1992 data, the enhanced network area in the fleld of view has twice the flux density, $10 \%$ higher supergranular velocity and 20\% larger cell sizes than the quiet, unenhanced network area. Based on the number densities and flow velocities of IN fields derived in this paper and a previous paper (Wang et al., 1995), we estimate that the lower limit of total energy released from the recycling of IN fields is $1.2 \times 10^{28} \mathrm{erg} \mathrm{s}^{-1}$, which is comparable to the energy required for coronal heating.
\end{abstract}

\section{Introduction}

The supergranule velocity pattern was first discovered by Hart $(1954,1956)$. It was first named and studied extensively by Leighton, Noyes, and Simon (1962) and Simon and Leighton (1964). They described it as a cellular convective motion well correlated with the magnetic network. The interaction between magnetic fields on the Sun and the convective flows has been a fundamental topic in solar physics (Spruit, 1981; Parker, 1982; Schmidt, Simon, and Weiss, 1985). Magnetograph observations with high resolution and sensitivity are essential to help us to understand the magnetic fields in the quiet Sun, and associated flow motions.

Intranetwork (IN) magnetic fields were first observed by Livingston and Harvey (1975) at Kitt Peak. They were described as 'discrete elements' of mixed polarities in the 'interior to the network'. The flux elements are as small as $5 \times 10^{16} \mathrm{Mx}$, the lifetimes are 5 to $90 \mathrm{~min}$ and they have possible radial proper motions. In recent years, with the improvement of the videomagnetograph system at Big Bear Solar Observatory, progress has been made in studies of the IN fields morphology and dynamics (Livi, Wang, and Martin, 1985; Martin, 1984, 1988, 1990; Martin, Livi,

Solar Physics 165: 223-235, 1996.

(C) 1996 Kluwer Academic Publishers. Printed in Belgium. 
and Wang, 1985; Wang, 1988; Wang, Zirin, and Shi, 1985; Wang and Zirin, 1988; Zirin, 1985, 1987, 1993). More recently, with spectroscopic techniques, it has been determined that IN fields are likely weak fields (compared with the kilo-gauss network field) (Keller et al., 1994; Lin, 1995).

In our previous paper (Wang et al., 1995, hereafter referred to as Paper I), we described the flux distribution of quiet-Sun magnetic elements. We found that IN flux ranges from $10^{16} \mathrm{Mx}$ to $2 \times 10^{18} \mathrm{Mx}$, with a peak distribution at $6 \times 10^{16} \mathrm{Mx}$. They are essentially in mixed polarity form and contribute an average flux density of $1.65 \mathrm{G}$ on the Sun at any given time. In this paper, we will concentrate our study on the velocity pattern of the $\mathbb{I N}$ fields. Although a similar study was made by Wang and Zirin (1988), the data used here are much improved, and therefore the derived velocity fields have much lower noise level permitting us to obtain more quantitative results, including the divergence of flows.

\section{Observations}

Our study is based on two sets of high-quality videomagnetograms, one obtained on June 4, 1992, and the other on July 28, 1994, both are near the solar disk center. The quality of the June 4, 1992 data is slightly better than that of July 28, 1994. For both days, the magnetograms were acquired by integrating 4096 video frames (2048 pairs of left- and right-handed circular polarization images) then recorded in 16-bit memory. For the 1992 data set, the 7-hour sequence consists of 73 images with the average time resolution of $5.7 \mathrm{~min}$; for the 1994 data set, the 9-hr sequence consists of 150 images with the average time resolution of $3.6 \mathrm{~min}$. The images were registered by the maximum correlation technique. The pixel resolution is $0.63^{\prime \prime}$ in $X$-direction and $0.5^{\prime \prime}$ in $Y$-direction. As we have determined in Paper I, the detection limit of magnetic flux is $10^{16} \mathrm{Mx}$, spatial resolution is $2^{\prime \prime}$, and the sensitivity of apparent flux density is $2 \mathrm{G}$.

To increase the ratio of signal to noise further, we did a running average for the re-registered data sets. However. we have to determine that the time interval chosen for the running average is small enough to avoid the smearing of IN features due to their proper motions. If we average 4 frames for the July 28,1994 data, then each summed image spans $4 \times 3.6=14.4 \mathrm{~min}$. If an IN element moves at a maximum speed of $0.5 \mathrm{~km} \mathrm{~s}^{-1}$, then in $14.4 \mathrm{~min}$, it moves $0.6^{\prime \prime}$, which is below the $2^{\prime \prime}$ spatial resolution. So the 4-frame running average is a good choice for the July 28, 1994 data. Similarly, a 3-frame running average was performed for the June 4, 1992 data. A high-quality original magnetogram has been shown in Paper I, so we do not repeat it here. 


\section{Results}

\subsection{HORIZONTAL VELOCITIES}

The flows of the IN elements were derived vtith standard local correlation tracking techniques (November, 1986; Wang and Zirin, 1988). We chose a boxcar-shaped window with a size of $5^{\prime \prime}$, which is half the window size used in our earlier studies (Wang and Zirin, 1988). Figures 1 and 2 show the flow maps of local correlation trackings for the July 28, 1994 and June 4, 1992 data, respectively. The grayscale background shows the magnetograms averaged over the whole observing period, so the motion of the individual IN elements makes them disappear by smearing. In general, IN fields move radially from the network cell center towards the boundaries. The peak speed, which usually occurs halfway between the cell center and the boundaries, is $0.4 \mathrm{~km} \mathrm{~s}^{-1}$. The r.m.s. speed is about $0.25 \mathrm{~km} \mathrm{~s}^{-1}$. These speeds are smaller than the values derived from the Doppler measurements (peak speed: $0.5 \mathrm{~km} \mathrm{~s}^{-1}$; r.m.s. speed: $0.3 \mathrm{~km} \mathrm{~s}^{-1}$, see Wang, 1988). So the peak and r.m.s. velocities derived from local correlation tracking are lower limits of the true velocities of supergranules. The reason is obvious: there are certain areas on the quiet Sun which do not have observable magnetic fields at a given time; the local correlation tracking would find zero velocities for these windows in these time bins, while the true supergranular velocities are not zero. The correlation velocities are averaged over many time bins, so they are smaller than the true values.

Figures 3 and 4 show the divergence maps (contours) based on the velocities presented in Figures 1 and 2. The white contours represent the areas of divergence flow, and dark contours, convergence. Again, the background shows the averaged magnetograms of these two days. It is not difficult to note that the divergence areas avoid strong magnetic elements and convergence areas are concentrated in the strong network areas. This correlation is better than $90 \%$. In addition, from the magnetograph movies, we have identified the converging and diverging centers visual. These centers agree well with what we derived in local correlation tracking.

The flow patterns can be further demonstrated by cork movies. The corks (testing particles) are initially uniformly distributed in the field of view and then let go under the local velocities derived by the local correlation tracking. Figures 5 and 6 show the cork distribution after 15 hours for the July 28, 1994 and June 4, 1992 regions, respectively. The background shows the averaged magnetogram again, but with the inverse absolute value of the flux density. The corks obviously outline the network very well after 15 hours, which is the typical time that an element takes to move from the cell center to the boundary. Figure 7 demonstrates a sequence of cork images for a selected $100^{\prime \prime}$ by $100^{\prime \prime}$ field of view from the July 28 , 1994 observation. The images show the cork locations after 3, 9, 19, 91, 27, and 33 hours. Two components of flows are clearly demonstrated in the series. The dominant component is a general radial flow pattern which sweeps intranetwork 


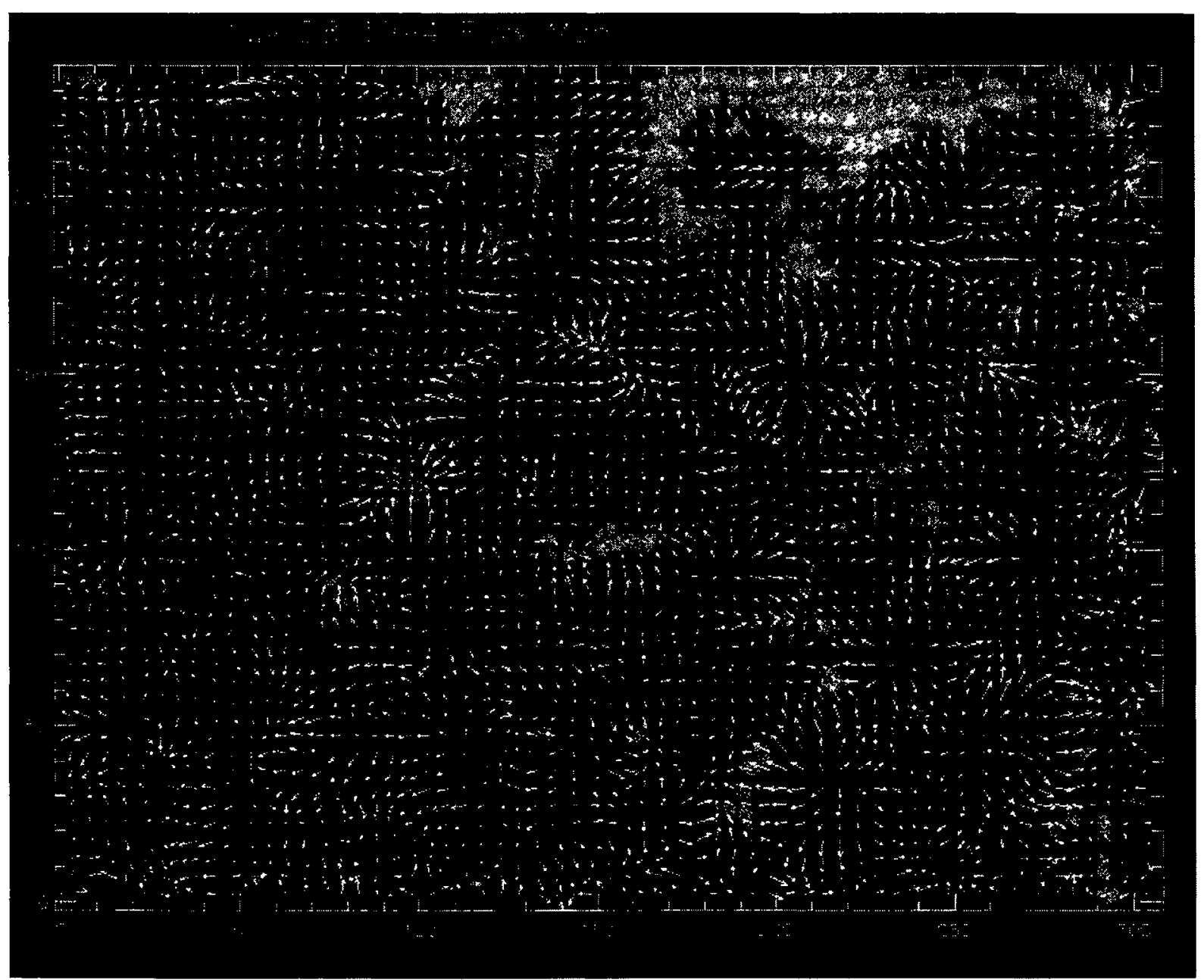

Fig. 1. The flow maps derived by local correlation tracking for the July 28, 1994 quiet-Sun magnetograms. The background gray-scale map is the average magnetogram for the entire period of tracking. The length of the longest arrows represents a speed on the order of $0.5 \mathrm{~km} \mathrm{~s}^{-1}$. The numbers labeled in the $X$-and $Y$-axes are in the units of arc sec. North is down and east is right.

fields to the boundary of the network. This component is easily visible in the flow maps (Figures 1 and 2). At $T=9$ hours (Figure 7), the cellular network pattern is roughly formed. The order of magnitude of this radial component (r.m.s. speed) is $0.25 \mathrm{~km} \mathrm{~s}^{-1}$. The second component is also obviously shown in the cork movies by the frames after $T=9$ hours. This secondary flow component moves along the network boundaries, and converges toward network magnetic elements. The typical speed is $0.05 \mathrm{~km} \mathrm{~s}^{-1}$. This second component is likely responsible for the concentration of magnetic elements to isolated patches. The same two components of motion were found by the tracking of granules (Title et al., 1990). Please note that the purpose of extrapolating the cork movie to over 30 hours is to clearly demonstrate the second velocity component; our observations do not cover such a long period. Viewing of the magnetograph movies and comparing them with flow maps show evidence that the first, flow component completely describes the 


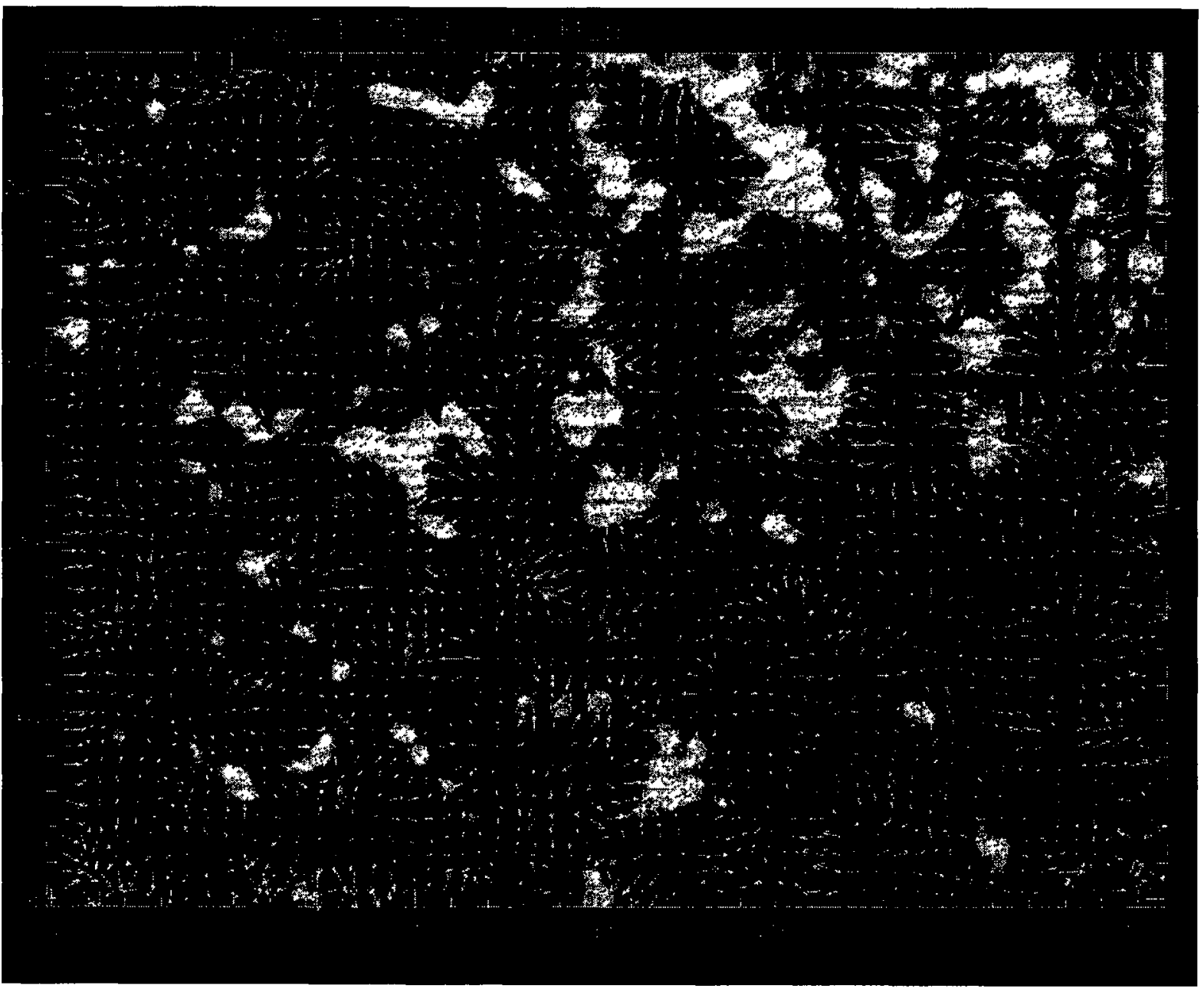

Fig. 2. The velocity pattern derived by local correlation tracking for the June 4, 1992 quiet-Sun magnetograms.

motion of IN fields, while the second component mainly describes the motion of network fields.

\subsection{VERTICAL VELOCITIES}

November (1994) discussed the relationship between the vertical velocity, $u_{z}$, and horizontal velocity, $\mathbf{u}_{h}$, of supergranules:

$$
u_{z}=h \nabla \cdot \mathbf{u}_{h},
$$

where $h$ is an effective scale height, and is essentially the photospheric density scale height, i.e., $\sim 150 \mathrm{~km}$, and $\nabla \cdot \mathbf{u}_{h}$ is the divergence we derived. Note that the above equation is valid when the observing region is not far away from the disk center. For both regions described in this paper, this approximation is acceptable, because the targets are within $15^{\circ}$ from the disk center. Because of the above relationship, Figures 3 and 4 also represent the vertical velocities of the 


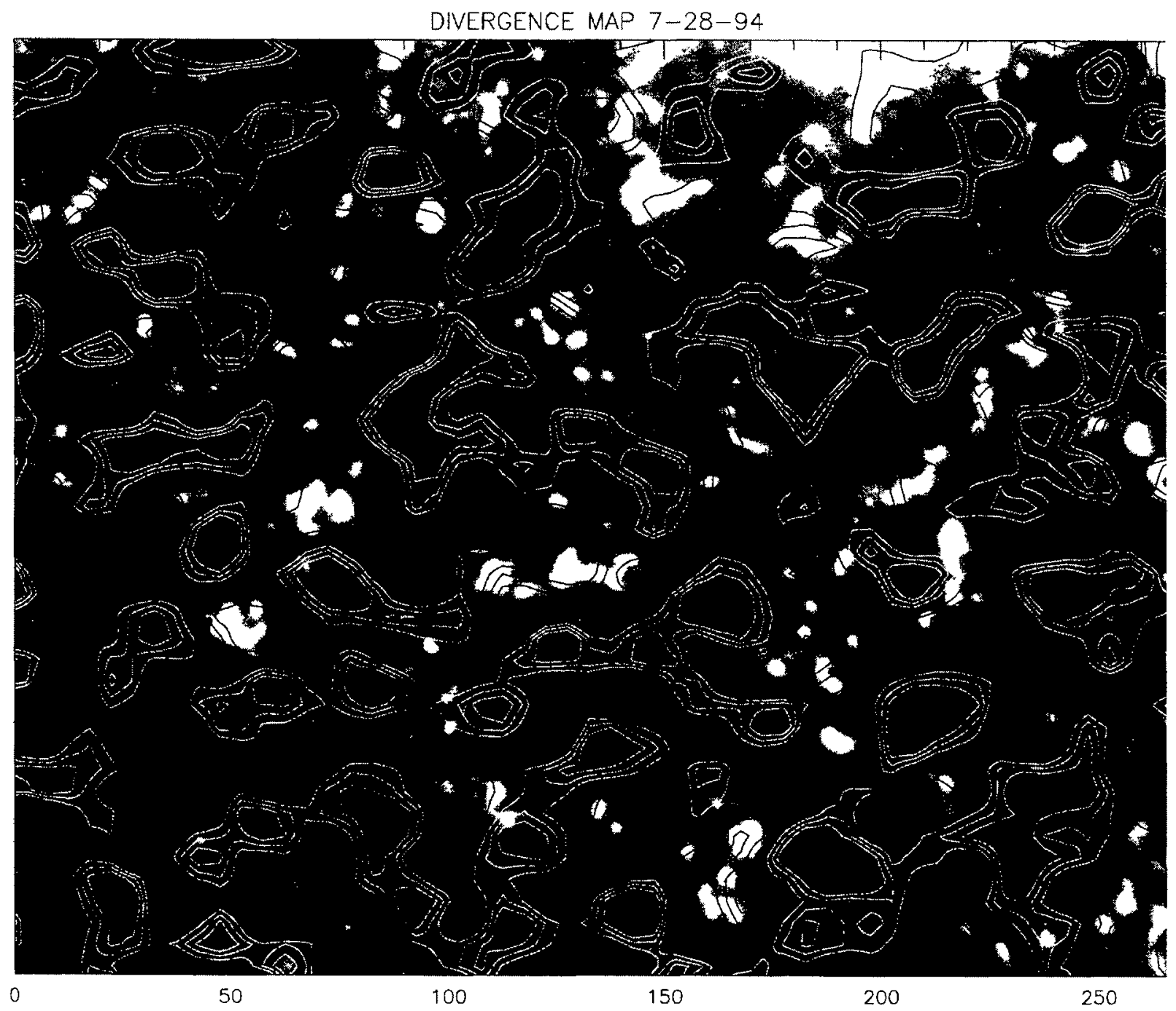

Fig. 3. The divergence map for the velocity fields of the July 28,1994 region. The dark contours are converging areas and white contours are diverging areas. Contour levels are $0.8,1.6$, and $2.4 \times 10^{-4} \mathrm{~s}^{-1}$. The gray-scale background is the averaged magnetogram.

supergranules - diverging areas correspond to upflow, and converging, downflow. The peak upflow and downflow speeds derived this way are around $50 \mathrm{~m} \mathrm{~s}^{-1}$, which explains the difficulty of Doppler observations for supergranule vertical velocities. For the same reason that we underestimated the horizontal velocity, we have also underestimated the amplitude of the vertical velocity. The convection flows do not seem to be isotropic; stronger downflows are correlated with stronger magnetic elements. The downflows corresponding to the strong magnetic field areas derived here are the true velocities, and are not caused by the Stokes $V$ asymmetry in the Doppler measurements, as discussed by Stenflo et al. (1984). 


\section{DIVERGENCE MAP $6-04-92$}

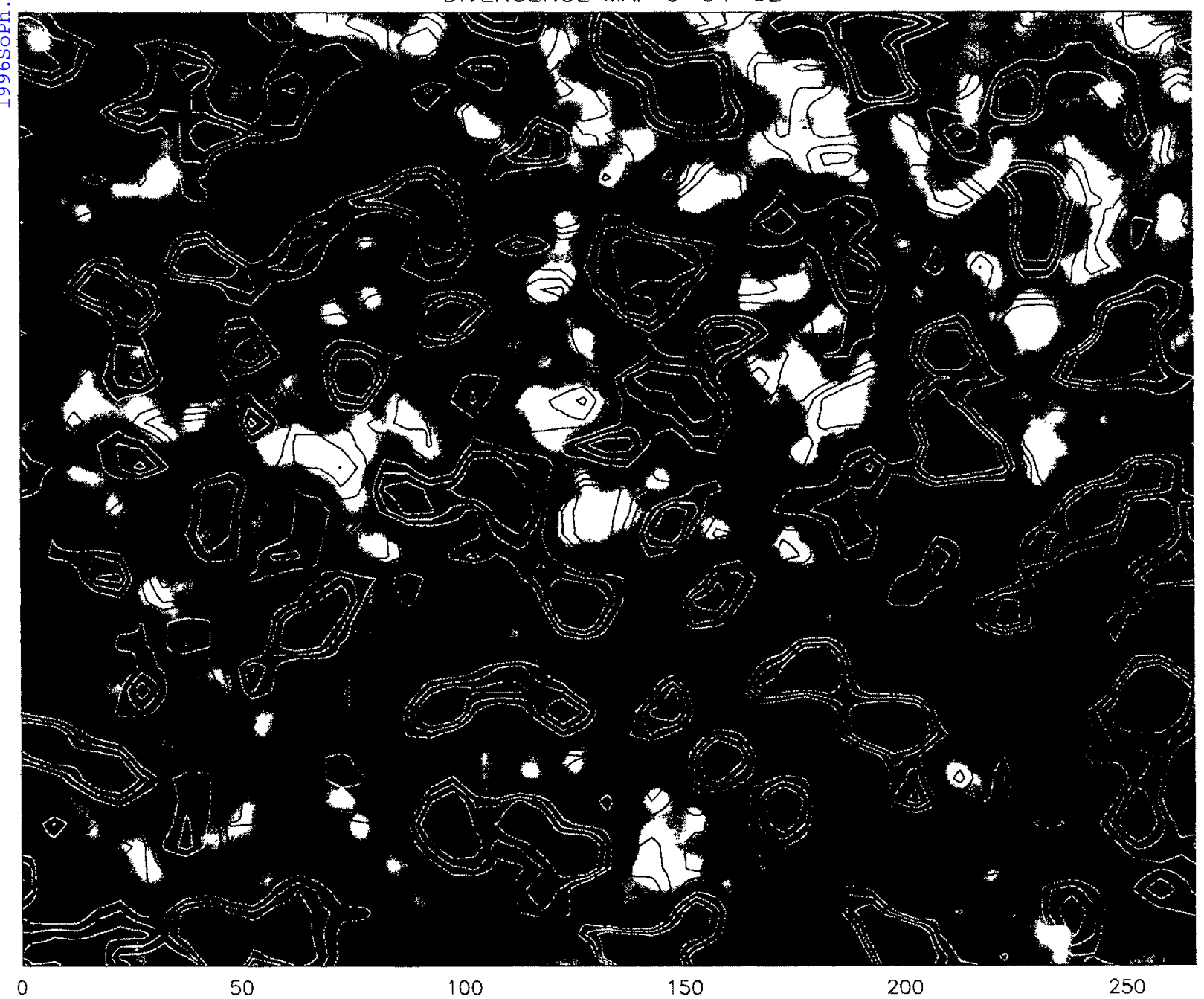

Fig. 4. The divergence map for the June 4, 1992 region.

\subsection{ENHANCED AND QUIET NETWORK}

The interaction between convection and magnetic fields is a fundamental and interesting problem. It has been found that granular convection motions have been substantially reduced in the area of strong magnetic field areas (Title, Yopka, and Tarbell, 1992). What are the properties of the supergranule cells for different magnetic activity levels? Wang (1988) studied the Dopplergrams measured at different magnetic levels, where he found that the variations of properties of supergranules were within uncertainties. However, the Dopplergrams were taken on different days. Any scale calibration or velocity calibration error could make the results difficult to interpret. In our June 4, 1992 field of view, the magnetic fields are stronger in the right half of the image. Since the images on the two halves were obtained in the same seeing condition, it provides a good opportunity to see if the properties of supergranules depend on the network magnetic fluxes. In Table I we list the key parameters which were derived from the June 4, 1992 observations. 


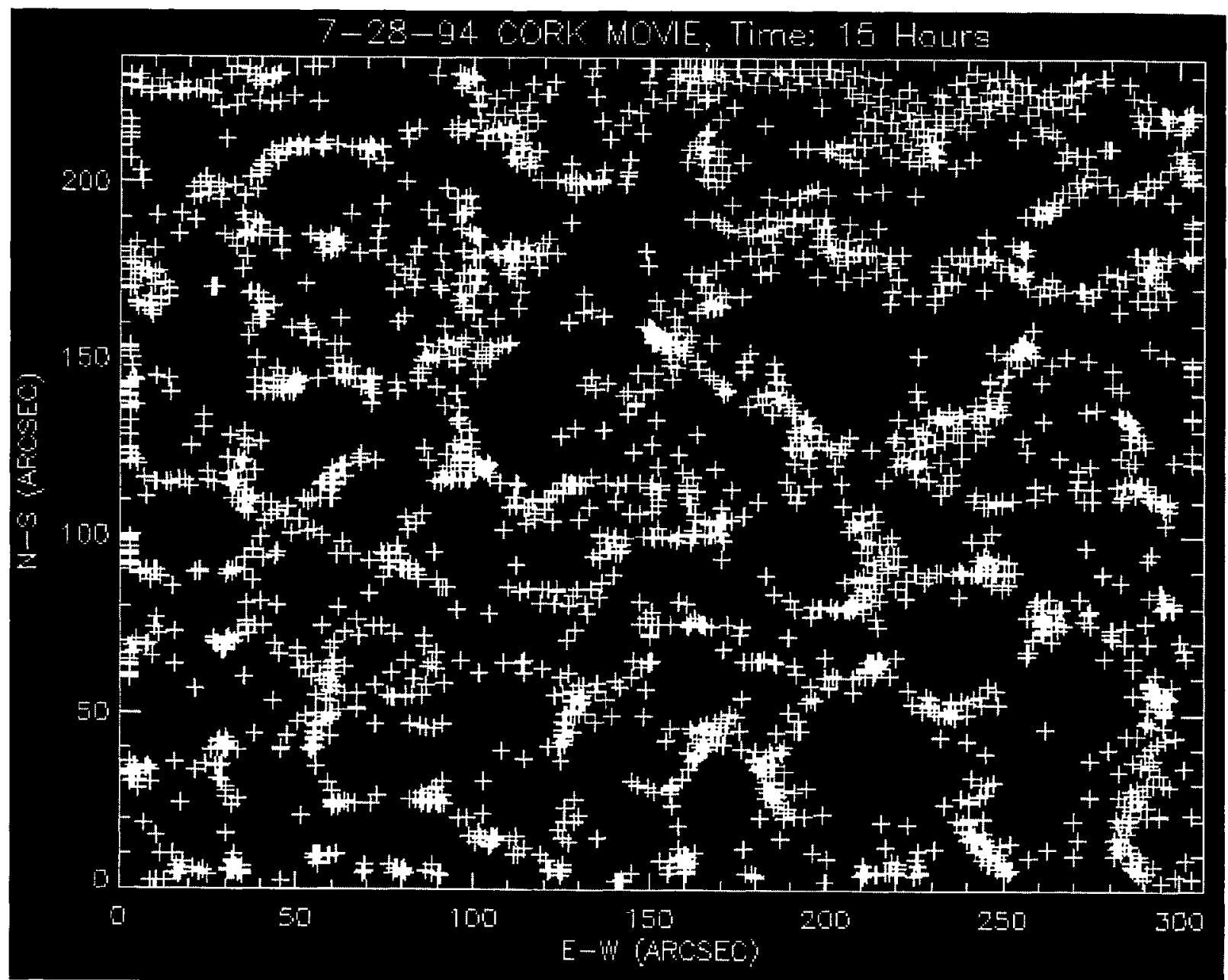

Fig. 5. The position of corks after 15 hours motion under the local velocities for the July 28,1994 region. The background is the average magnetogram, but is a negative image of the absolute value of flux density.

TABLE I

Properties of supergranules

\begin{tabular}{lll}
\hline & Quiet fields (left) & Enhanced fields (right) \\
\hline Mean flux density & $7.9 \mathrm{G}$ & $14.0 \mathrm{G}$ \\
R.m.s. velocities & $0.934 \mathrm{~km} \mathrm{~s}^{-1}$ & $0.250 \mathrm{~km} \mathrm{~s}^{-1}$ \\
R.m.s. divergence & $2.40 \times 10^{-4} \mathrm{~s}^{-1}$ & $2.66 \times 10^{-4} \mathrm{~s}^{-1}$ \\
Cell size & $23000 \mathrm{~km}$ & $28000 \mathrm{~km}$ \\
\hline
\end{tabular}

The cell size was derived from the auto-correlation curve on the divergence map in the $Y$-direction. Since the subwindows were elongated in the $Y$-direction, the $X$-direction has too few samples of supergranular cells to make accurate autocorrelation measurements. The distance between the main peak and the second 


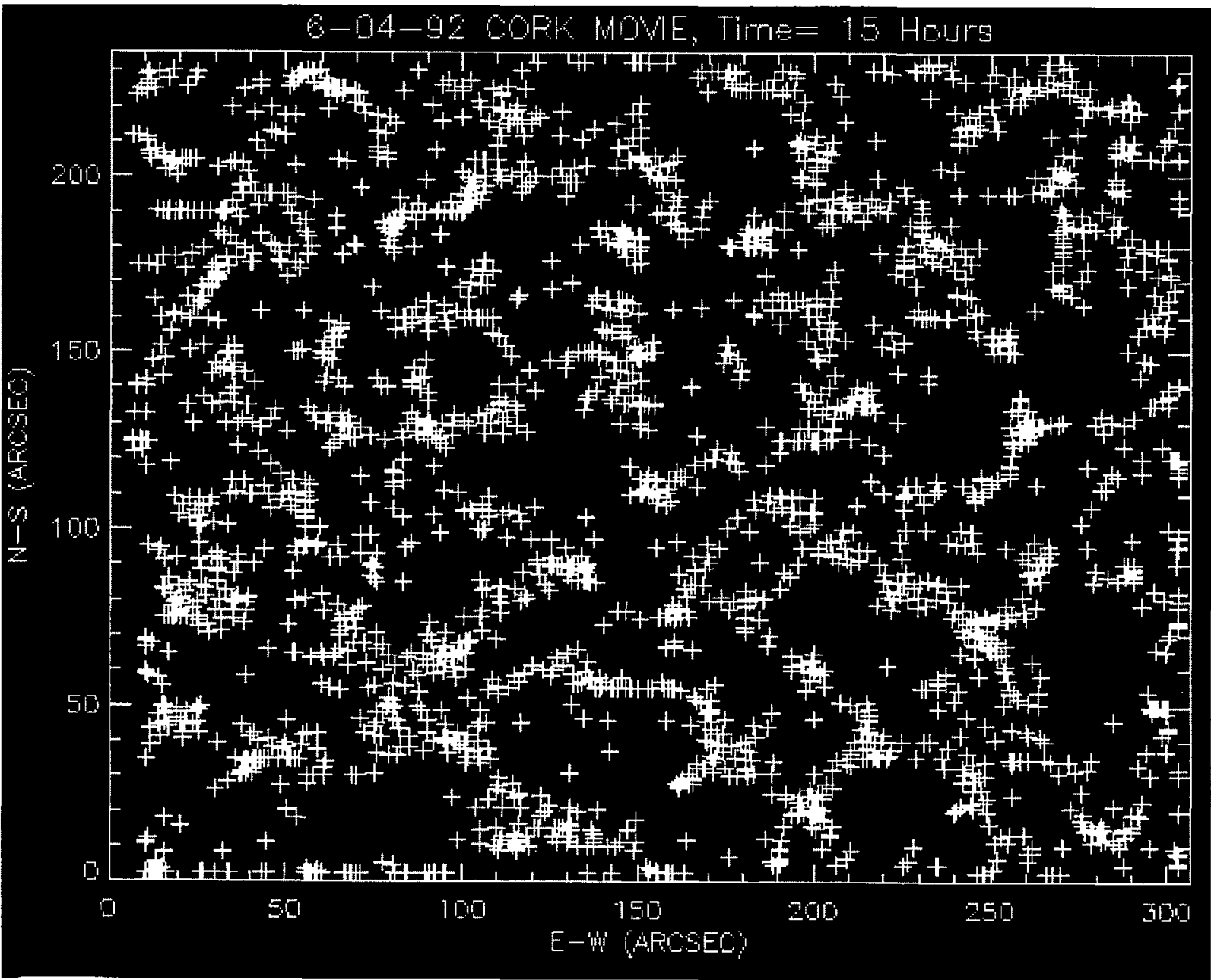

Fig. 6. The position of corks after 15 hours motion under the local velocities for the June 4, 1992 region.

peak in an auto-correlation curve shows the mean size of the cells (Simon and Leighton, 1964). The auto-correlation curves are plotted in Figure 8. The bottom panel is for the right half (enhanced fields) and the top panel is for the left half (quiet fields) of the field of view.

We find the enhanced sub-window has two times the flux density, $10 \%$ stronger horizontal r.m.s. velocity and $20 \%$ larger cell size, compared to the quiet subwindow. The physical nature of the differences is unclear. The variation of the cell size is qualitatively consistent with the finding of Komm, Howard, and Harvey (1995), where they concluded that the supergranular cell size increases as magnetic activity increases.

\subsection{INTERACTION OF MAGNETIC ELEMENTS}

In Paper I, we described the nature of the mixed polarities of IN fields and derived the mean number density and the flux of IN elements. When an IN element is pushed into the network, if it has the same magnetic polarity as the network element, the 


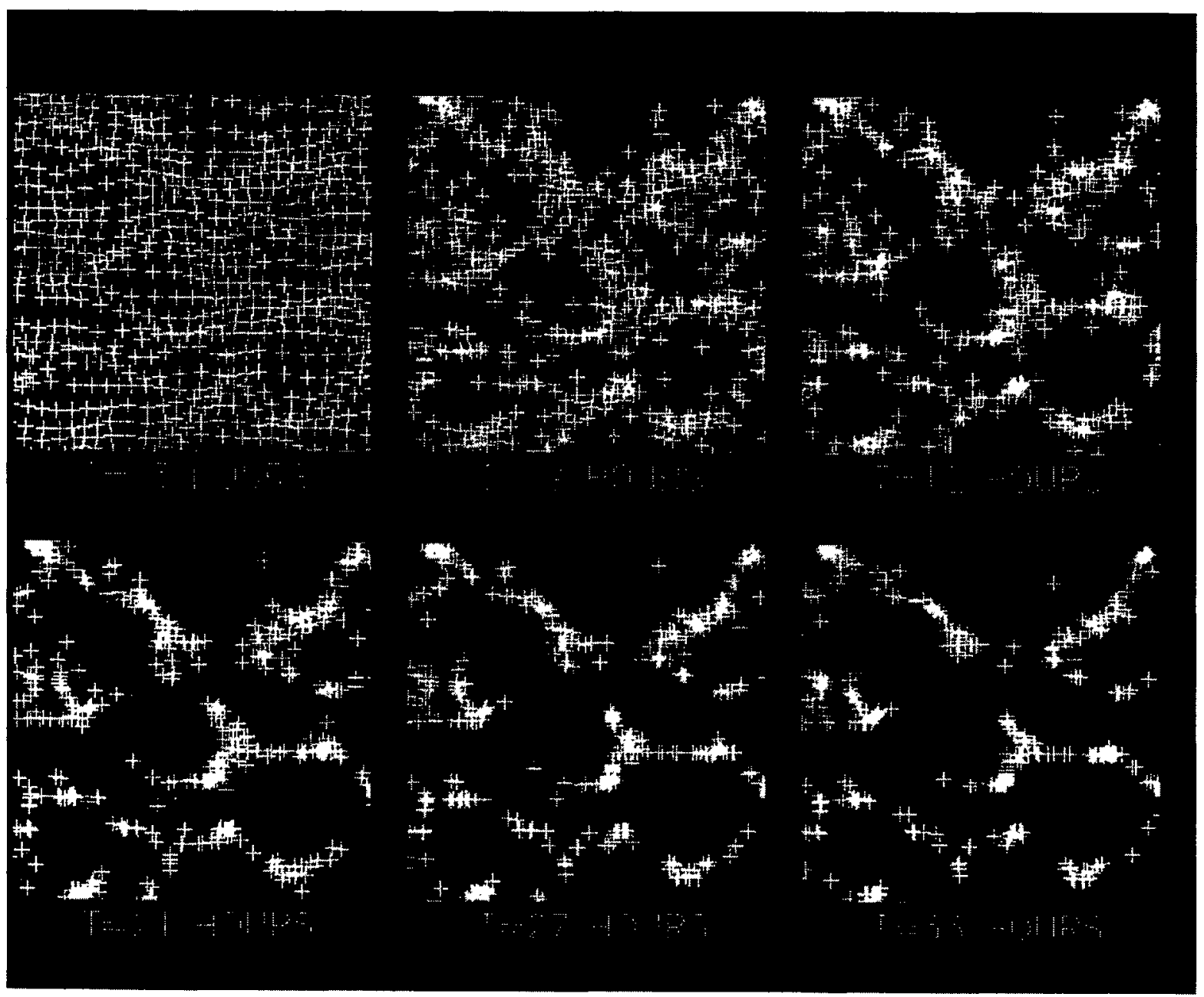

Fig. 7. A sequence of cork images for a sub-window in the July 28,1994 region. The field of view is $100^{\prime \prime}$ by $100^{\prime \prime}$.

IN element will simply merge with network, no energy will be released; if it has opposite polarity, it will 'cancel' with the network element, likely by slow magnetic reconnection. Magnetic energy will be released due to the reconnection. Now we would like to estimate the amount of the energy which may be released in IN-network encounters.

From the movies, we have noted that most IN elements disappear when they each the network. However, we have to admit that the seeing variation makes the identification difficult. Suppose IN elements move at an average speed of $0.3 \mathrm{~km} \mathrm{~s}^{-1}$, the diameter of supergranular cell is $39000 \mathrm{~km}$, and IN elements emerge in the inner $\frac{1}{2}$ radius of the cells. Then on average, it takes $T=11$ hours for the elements to get to the network boundary. This value can be estimated as the upper limit of the lifetime of $\mathbb{I N}$ elements, since an $\mathbf{I N}$ element may disappear before it reaches the network boundary. In Paper I, we found that the mean number density of IN fields is 3.5 per $100 \operatorname{arcsec}^{2}$, i.e., $N=4.05 \times 10^{5}$ over the whole Sun. This value $N$ is a lower limit, because of the limit of sensitivity. The replenishment 

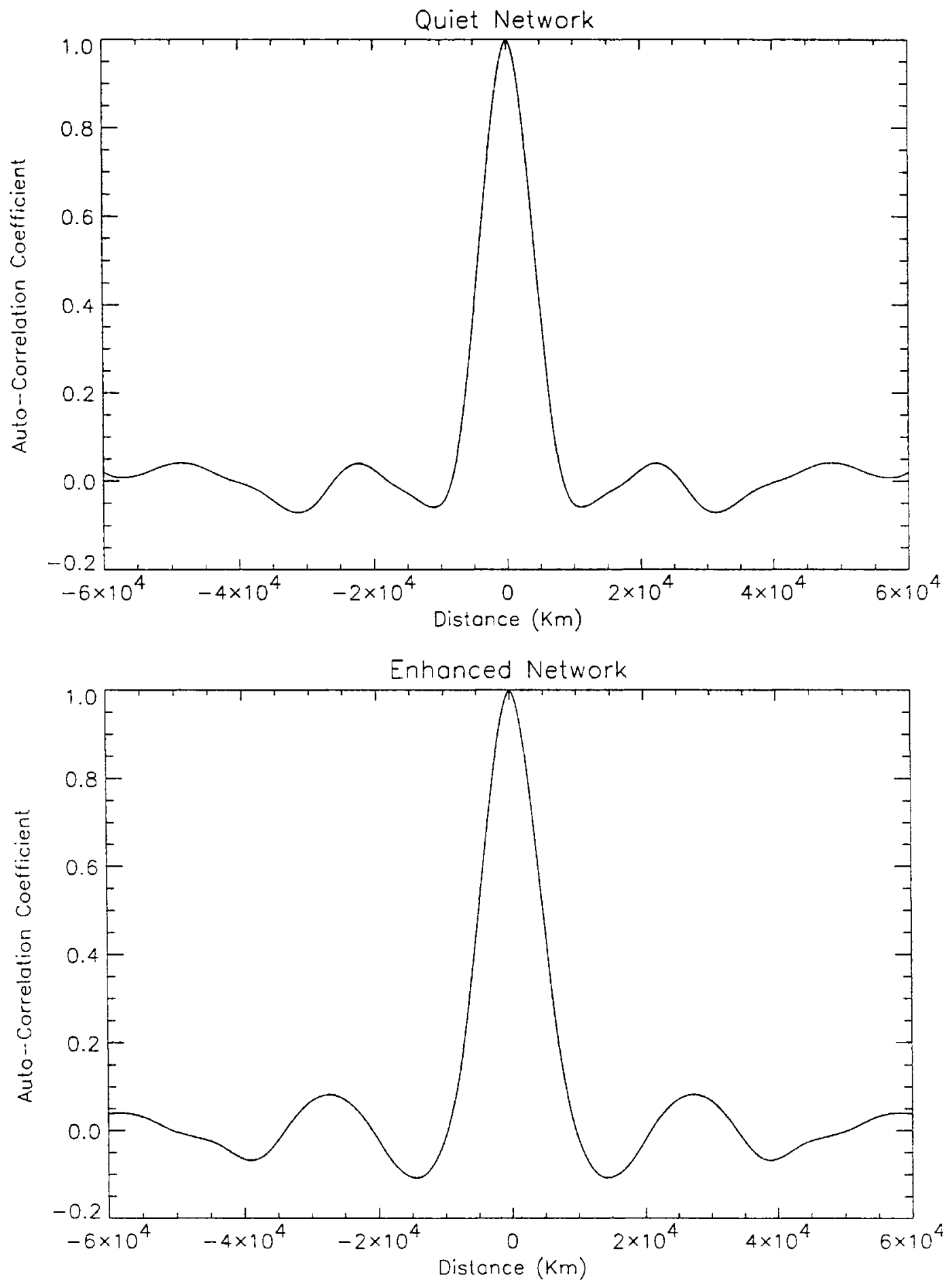

Fig. 8. The vertical auto-correlation curves of the divergence map for the two sub-windows in the June 4, 1992 region.

rate of IN fields is then $R=N / T=10.2$ elements $\mathrm{s}^{-1}$. The energy dissipation rate can be estimated as

$$
\frac{\mathrm{d} E}{\mathrm{~d} t}=\frac{B^{2}}{8 \pi} R V=B \frac{F}{8 \pi} R H
$$


where $V$ is the volume and $H$ is the depth of an $\mathrm{IN}$ element, and $F$ is the mean magnetic flux of $\mathbb{I N}$ elements. We adopt $H$ as the scale height of horizontal flows, which was found to be $2400 \mathrm{~km}$ (November, 1994), $B=500 \mathrm{G}$ (Lin, 1995; Keller et al., 1994), and $F=2.5 \times 10^{17} \mathrm{Mx}$ (Paper I). We then obtain $\mathrm{d} E / \mathrm{d} t=$ $1.2 \times 10^{28} \mathrm{ergs} \mathrm{s}^{-1}$. This number is comparable to the energy rate which is required for the coronal heating, i.e., $1.8 \times 10^{28} \mathrm{ergs} \mathrm{s}^{-1}$ (Withbroe and Noyes, 1977). Since $T$ is an upper limit and $N$ is a lower limit, the output value $\mathrm{d} E / \mathrm{d} t$ is a lower limit.

\section{Summary}

We applied local correlation tracking techniques to detect the flows of magnetic fields in the quiet Sun, and obtain the following results:

(1) There are two cormponents in the flows: radial flows which move IN fields from network center to the boundaries, and lateral flows which move along the network boundaries and converge toward the strong magnetic elements.

(2) We constructed divergence maps based on horizonal velocities and derived the vertical velocity of supergranules. The peak vertical velocity is only $50 \mathrm{~m} \mathrm{~s}^{-1}$.

(3) In the June 4, 1992 data, the left and right halves in the observing field of view have different levels of magnetic activity. The mean flux density on the right side is about twice of that on the left side. The stronger magnetic area has $10 \%$ larger supergranular velocities, and $20 \%$ larger cell sizes.

(4) We estimated that the total energy which could be released from the recycling of IN fields is at least $1.2 \times 10^{28} \mathrm{ergs} \mathrm{s}^{-1}$, which is comparable to the energy required for coronal heating.

\section{Acknowledgements}

The operation of BBSO was supported by NSF grant ATM-9122023 and by NASA grant NAGW-1979. HW has been supported by NASA grant NAG5-2090 and NJIT SBR funding.

\section{References}

Hart, A. B.: 1954, Monthly Notices Roy. Astron. Soc. 114, 9.

Hart, A. B.: 1956, Monthly Notices Roy. Astron. Soc. 116, 38.

Keller, C. J., Deubner, F.-L., Egger, U., Fleck, B., and Povel, H. P.: 1994, Astron. Astrophys. 286, 696.

Komm, R. W., Howard, R. F., and Harvey, J. W.: 1995, Solar Phys. 158, 213.

Leighton, R. B., Noyes, R. W., and Simon, G. W.: 1969, Astrophys. J. 135, 474.

Lin, H.: 1995, Astrophys. J. 446, 421.

Livi, S. H. B., Wang, J., and Martin, S. F.: 1985, Australian J. Phys. 38, 855.

Livingston, W. C. and Harvey, J.: 1975, Am. Astron. Soc. Bull. 7, 346. 
Martin, S.F.: 1984, in S. Keil (ed.), Small-Scale Dynamical Process in Quiet Stellar Atmospheres, p. 30.

Martin, S. F.: 1988, Solar Phys. 117, 243.

Martin, S.F.: 1990, in J. O. Stenflo (ed.), Solar Photosphere: Structure, Convection and Magnetic Fields, p. 129.

Martin, S. F., Livi, S. H. B., and Wang, J.: 1985, Australian J. Phys. 38, 929.

November, L.: 1986, Appl. Optics 25, 392.

November, L.: 1994, Solar Phys. 154, 1.

Parker, E. N.: 1982, Astrophys. J. 256, 746.

Schmidt, H. U., Simon, G. W., and Weiss, N. O.: 1985, Astron. Astrophys. 148, 191.

Simon, G. W. and Leighton, R. B.: 1964, Astrophys. J. 140, 1120.

Spruit, H. C.: 1981, Astron. Astrophys. 98, 155.

Stenflo, J. O., Harvey, J. W., Brault, J. W., and Solanki, S.: 1984, Astron. Astrophys. 131, 333.

Title, A. M., Topka, K. P., and Tarbell, T. D.: 1992, Astrophys. J. 393, 782.

Title, A. M., Shine, R. A., Tarbell, T. D., Topka, K. P., and Sharmer, G. B.: 1990, IAU Symp. 138, 49. Wang, H.: 1988, Solar Phys. 117, 343.

Wang, H. and Zirin, H.: 1988, Solar Phys. 115, 205.

Wang, J., Zirin, H., and Shi, Z.: 1985, Solar Phys. 98, 241.

Wang, J., Wang, H., Tang, F., Lee, J., and Zirin, H.: 1995, Solar Phys. 160, 277 (Paper I).

Withbroe, G. L. and Noyes, R. W.: 1977, Ann. Rev. Astron. Astrophys. 15, 363.

Zirin, H.: 1985, Australian J. Phys. 38, 961.

Zirin, H.: 1987, Solar Phys. 110, 101.

Zirin, H.: 1993, in H. Zirin, G. Ai, and J. Wang (eds.), Magnetic and Velocity Fields of Solar Active Regions, p. 215. 